\title{
Constructing a small category of setoids
}

\author{
K.O. Wilander
}

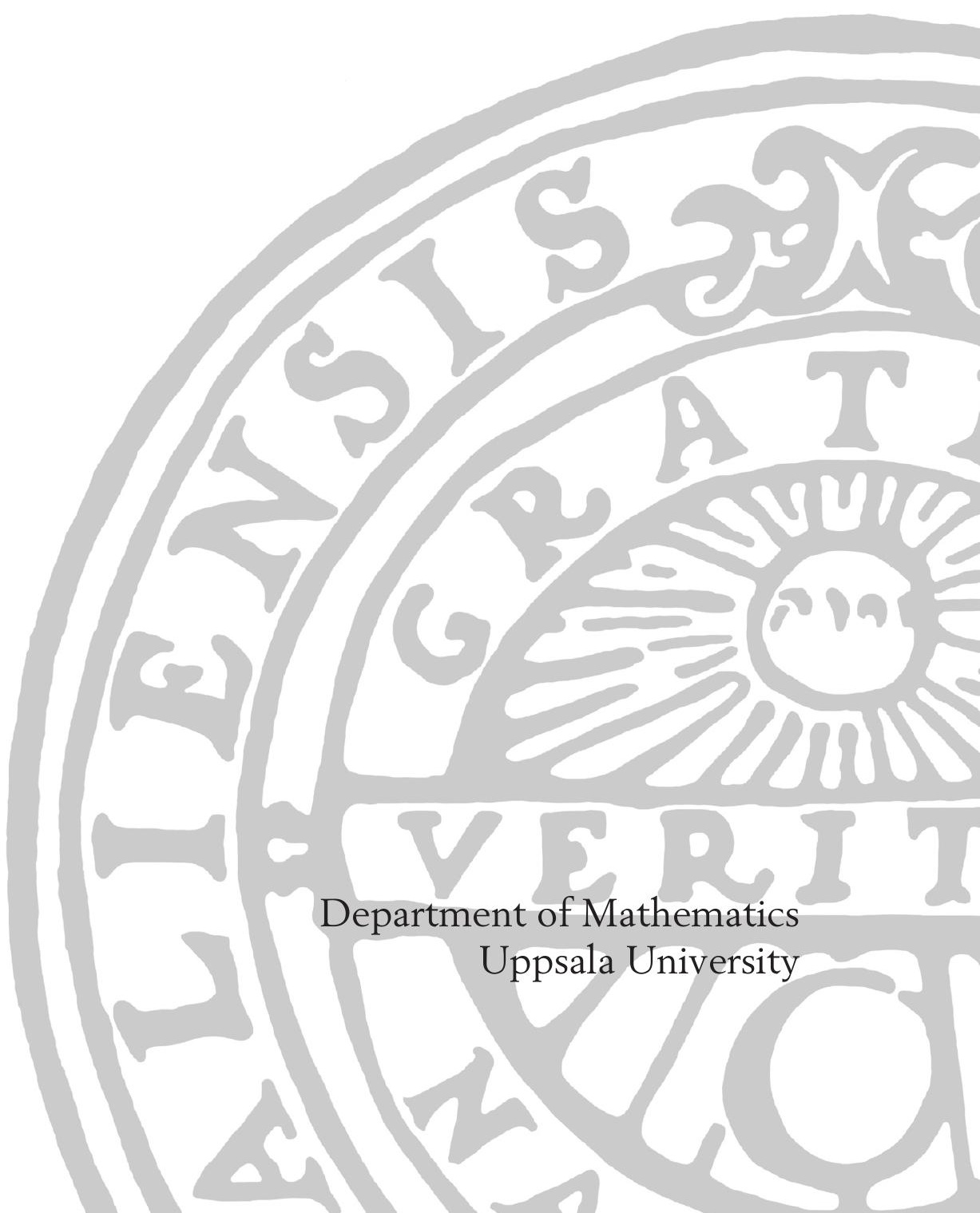





\title{
CONSTRUCTING A SMALL CATEGORY OF SETOIDS
}

\author{
K.O. WILANDER
}

\begin{abstract}
Consider the first order theory of a category. It has a sort of objects, and a sort of arrows (so we may think of it as a small category). It is shown that, assuming the principle of unique substitutions, the setoids inside a type theoretic universe provide a model for this first order theory. It is also shown that the principle of unique substitutions is not derivable in type theory, but that it is strictly weaker than the principle of unique identity proofs.
\end{abstract}

In this note the construction of a small category of setoids is considered. A small category has a set of objects and a set of all arrows, and can be described in first order logic with equality. This differs from the usual type-theoretical formalisation of a category, which has a type of objects and a set of arrows for each pair of objects (so might be considered locally small). This usual formalisation does not use a (propositional) equality on the objects of the category, instead relying on dependent typing and typechecking, particularly for the formalisation of the composition of arrows. In contrast, the formalisation presented in this note does include both an equality on objects and an equality on arrows making all arrows comparable (not just those given with the same domain and codomain), and requires all constructions to respect these equalities.

A setoid is a constructive counterpart of a set, and consists of a type (or a set) together with an equivalence relation on this type, and the maps of setoids are functions respecting these equivalence relations.

We will also need a notion of smallness for types, which is provided by the notion of a universe, and from a classical set-theoretic perspective corresponds to an initial segment $V_{\kappa}$ of the cumulative hierarchy, with $\kappa$ strongly inaccessible. This is needed, not only to make the collection of small setoids a type, but also to let us introduce a propositional equality on setoids.

The final ingredient is an extra axiom for the identity type on a universe, related to the auxiliary eliminator K suggested by Streicher [13], and in its full form equivalent to the uniqueness-of-identity-proofs-principle UIP. This axiom

$$
(\mathrm{US}-\operatorname{refl}(U)) \quad X: U, \alpha: \mathrm{Id}_{U}(X, X), x: T(X) \vdash \operatorname{Id}_{T(X)}\left(x, \operatorname{subst}_{\alpha}(x)\right)
$$

(for a universe $U$ with decoding family $T$ ) expresses that substitution along any reflexivity proof is the identity, and so agrees (pointwise) with substitution along the canonical proof of reflexivity, or more simply, that there is a unique substitution on every $U$-set. It is needed to avoid problems related to the non-uniqueness of identity proofs $[6]$.

We begin with a short reminder of the axioms of a category in the appropriate form, followed by a short discussion of the appropriate notion of model of such a

Date: February 11, 2011. 
theory. Then follows the model construction, in stages, followed by a discussion of why the extra axiom is needed.

\section{The AXIOMs of a SMALl CATEGory}

A small category is, in the notation of [8], [7, B2.3.1], [11], a three-sorted structure with sorts $C_{0}, C_{1}$, and $C_{2}$ (to be understood as the sorts of objects, arrows, and composable arrows), with six function symbols (and their arities)

$$
\begin{array}{lll}
\text { id : } C_{0} \rightarrow C_{1} & \text { dom: } C_{1} \rightarrow C_{0} & \text { cod: } C_{1} \rightarrow C_{0} \\
\text { comp : } C_{2} \rightarrow C_{1} & \text { fst : } C_{2} \rightarrow C_{1} & \text { snd : } C_{2} \rightarrow C_{1},
\end{array}
$$

to be understood as giving, in turn, the identity arrow on an object, the domain and codomain of an arrow, the composite of a composable pair of arrows, and the first and second arrow in a composable pair. Before giving the axioms the structure should satisfy, we introduce two shorthand notations:

$$
\begin{aligned}
h \sim g \circ f & \equiv\left(\exists x \in C_{2}\right)(\operatorname{fst}(x)=f \& \operatorname{snd}(x)=g \& \operatorname{comp}(x)=h) \\
k \circ h \sim g \circ f & \equiv\left(\exists m \in C_{1}\right)(m \sim k \circ h \& m \sim g \circ f) .
\end{aligned}
$$

The axioms of a category are then:

(1) $\operatorname{dom}(\operatorname{id}(x))=x$

(2) $\operatorname{cod}(\mathrm{id}(x))=x$

(3) $\operatorname{dom}(\operatorname{comp}(u))=\operatorname{dom}(\operatorname{fst}(u))$

(4) $\operatorname{cod}(\operatorname{comp}(u))=\operatorname{cod}(\operatorname{snd}(u))$

(5) $\operatorname{fst}(u)=\operatorname{fst}(v) \& \operatorname{snd}(u)=\operatorname{snd}(v) \longrightarrow u=v$

(6) $\operatorname{dom}(f)=\operatorname{cod}(g) \longrightarrow\left(\exists u \in C_{2}\right)(\mathrm{fst}(u)=g \& \operatorname{snd}(u)=f)$

(7) $f \sim f \circ \mathrm{id}(\operatorname{dom}(f))$

(8) $f \sim \mathrm{id}(\operatorname{cod}(f)) \circ f$

(9) $k \sim f \circ g \& l \sim g \circ h \longrightarrow k \circ h \sim f \circ l$

\section{Models IN TYPe THEORY}

There are several possible notions of a type-theoretical model for (many-sorted) first order theories of this kind. Considering our example:

- The simplest one is insisting that all equalities be definitional, or in other words, that the left- and right-hand sides compute to the same thing. This is what Per Martin-Löf calls an intensional category. Though convenient for many purposes (most particularly for work on internal models of type theory) it is not well suited for the present purposes, since the equality of setoid maps is not the definitional equality. It may also be quite difficult to construct intensional categories, and frequently the objects of study become contexts (possibly of particular form) and associated context morphisms.

- We can demand definitional equality on some of the sorts, but a (necessarily coarser) propositional equality on the others. While an approach of this kind will frequently work well, there is an important associated problem, namely that all functions must respect equality. If the equality on the codomain is definitional, but that on the domain is propositional, it will generally be difficult or impossible to solve this problem (which may be thought of as a kind of coherence problem). Having some of the equalities definitional (thus without proof objects) and other equalities propositional (thus with proof objects) may also in itself be inconvenient. 
- It is possible to use the standard notion of equality in type theory, namely the identity type, as interpretation of all equalities. The identity type is intended as an internalisation of the definitional equality, and working in this way is at the base of direct formalisation of mathematics into type theory. If one can consistently stay with this interpretation, there will also be no coherence problems showing up. But type theory is a fundamentally intensional system, and this creates problems. For our purpose, the problem is again the equality of setoid maps, which is extensional, identifying two functions if they agree at each argument. Having accepted this, this approach turns naturally into the next one:

- Bishop-style formalisation in type theory is based on Bishop's view of a set as consisting not only of a way to construct elements of the set, but also of a notion of when two elements are equal [1], [2]. This leads directly to the concept of a setoid, and is in fact the approach we will follow.

A setoid $\mathcal{A}$ consist of a type $A$ : Set together with an (infix) equivalence relation $=_{\mathcal{A}}:(A, A)$ Set on that type. That the relation is an equivalence relation is witnessed by terms $\operatorname{refl}_{\mathcal{A}}:(\Pi x: A)\left(x={ }_{\mathcal{A}} x\right), \operatorname{sym}_{\mathcal{A}}:(\Pi x, y: A)\left(x={ }_{\mathcal{A}} y \longrightarrow y={ }_{\mathcal{A}} x\right)$, and $\operatorname{trans}_{\mathcal{A}}:(\Pi x, y, z: A)\left(x=_{\mathcal{A}} y \& y={ }_{\mathcal{A}} z \longrightarrow x=_{\mathcal{A}} z\right)$ for reflexivity, symmetry, and transitivity respectively. Since these are the framework for our formalisation, and not objects of it, the notion of equality of setoids is not important (equality of sorts does not need an interpretation), so we may as well stick with definitional equality.

A map of setoids is an extensional function, that is, a function $f: \mathcal{A} \rightarrow \mathcal{B}$ is a function $A \rightarrow B$ on the underlying types, together with a proof

$$
\operatorname{ext}_{f}:(\Pi x, y: A)\left(x={ }_{\mathcal{A}} y \longrightarrow f(x)={ }_{\mathcal{B}} f(y)\right)
$$

that it respects the setoid equalities. Again, the equality of two such functions is not essential, since they are the interpretations of function symbols (which are never compared); however, two functions are considered equal if they agree pointwise, that is $f=g: \mathcal{A} \rightarrow \mathcal{B}$ if we can show that $(\forall x: A)\left(f(x)={ }_{\mathcal{B}} g(x)\right)$.

As indicated, we will interpret the sorts of a theory by setoids, (well-sorted) equalities by the setoid equalities, and the function symbols as setoid functions.

\section{TYPE THEORY, BACKGROUND AND NOTATION}

The type theory used is the predicative intensional version of Martin-Löf type theory, as described in [9], [10]. This formulation uses the so-called Logical Framework, with a type Set containing inductively defined types, and which is also the universe of propositions. The most important set formers are the $\Pi$-sets of dependent functions, which also interpret the universal quantifier, the $\Sigma$-sets of dependent pairs, which also interpret the existential quantifier, the empty set $\mathbf{N}_{0}$, which also interprets $\perp$, the always false statement, the natural numbers $\mathbf{N}$, and the (intensional) identity sets $\operatorname{Id}_{A}(x, y)$ (where $A$ : Set and $x, y: A$ ). From these, we may define the non-dependent function set $A \longrightarrow B$ as a $\Pi$-set with constant family, interpreting implication, and the product set $A \times B$ as a $\Sigma$-set with constant family, interpreting the conjunction. We also have the intuitionistic negation $\neg P$ defined as $P \longrightarrow \perp$. 
To this comes a universe (á la Tarski) $U$ : Set, which should be understood as a collection of names for other sets, together with a decoding family $T$ : $(U)$ Set interpreting each name. ${ }^{1}$ What particular sets have names in $U$ will not be important for this article, not even the standard assumptions that $U$ contains its own 'small' versions of both $\Sigma$ - and $\Pi$-sets are needed.

Finally, we will make much use of a derived eliminator subst ${ }_{\alpha, C}$ (defined in $[9$, Section 8.1]), which for an $\alpha$ : $\operatorname{ld}_{A}(x, y)$ is of type $C(x) \rightarrow C(y)$. We will write just subst $_{\alpha}$, rather than subst ${ }_{\alpha, T}$, when the family of sets is the decoding family for the universe.

\section{Setoids in $U$}

Since our aim is to construct a category of setoids, it is clear that the interpretation of the sort $C_{0}$ should be the setoids. However, the interpretation of the sort $C_{0}$ should also be $a$ setoid. For size reasons, it is clear that we can not have both, so we must construct a category of small setoids, with the universe $U$ providing the notion of smallness.

Transferring the definition of a setoid to the universe $U$, a $U$-setoid $\mathcal{A}$ consists of

- an element $A: U$ for the small underlying set,

- a function $=_{\mathcal{A}}: T(A) \rightarrow T(A) \rightarrow U$, which will be written in infix notation, for the equality relation,

- a proof refl $\mathcal{A}_{\mathcal{A}}:(\forall x, y: T(A)) T\left(x=_{\mathcal{A}} y\right)$ of reflexivity,

- a proof $\operatorname{sym}_{\mathcal{A}}:(\forall x, y: T(A))\left(T\left(x={ }_{\mathcal{A}} y\right) \longrightarrow T\left(y={ }_{\mathcal{A}} x\right)\right)$ of symmetry, and

- a proof trans $\mathcal{A}_{\mathcal{A}}:(\forall x, y, z: T(A))\left(T\left(x={ }_{\mathcal{A}} y\right) \& T\left(y={ }_{\mathcal{A}} z\right) \longrightarrow T\left(x={ }_{\mathcal{A}} z\right)\right)$, of transitivity of the equality relation.

Repeated use of the $\Sigma$ set former makes this a set. Note that this formulation has used a minimum of assumptions about the universe $U$; if $U$ contains for example implications, then $T(-) \longrightarrow T(-)$ could have been written as $T(-\longrightarrow-$ ) (where the arrow now is 'in $U$ ', but is interpreted as the standard implication by $T$ ), giving an equivalent notation with fewer occurrences of the decoding family $T$.

Next is the question of the appropriate equality to turn this set into a setoid. Since the equality must be propositional, the definitional equality is no longer an option, but there are many possible choices. The choice for the purposes of this note is: a proof that two $U$-setoids $\mathcal{A}$ and $\mathcal{B}$ are equal consists of

(1) a proof $\alpha: \operatorname{ld}_{U}(A, B)$, that the (names of the) underlying small sets are equal, and

(2) a proof of $(\forall x, y: T(A))\left(x=\mathcal{A} y \longleftrightarrow \operatorname{subst}_{\alpha}(x)={ }_{\mathcal{B}} \operatorname{subst}_{\alpha}(y)\right)$, that two elements are equal in $\mathcal{A}$ if and only if they are equal in $\mathcal{B}$ (or rather, their images under substitution are).

(So the equality is a $\Sigma$-set or, viewed logically, an existential statement.)

There are several reasons for choosing this equality. The simplest reason is that it is easy to work with: directly reflecting the structure of the $U$-setoids themselves it is conceptually simple, and (reasonably) easy to work with. It also separates data from logic, and while this might not be entirely natural in a system as strictly

\footnotetext{
${ }^{1}$ The type Set is not a universe in this sense, even though it too might be thought of as containing names for (small) types. The differences are somewhat technical, and the interested reader may consult [3].
} 
propositions-as-types as Martin-Löf type theory, we can then consider a similar construction in other systems without this strong identification. Finally, it introduces a very slight bit of proof independence, which may be exploitable for interpreting extra function symbols, corresponding to chosen categorical constructions (as opposed to constructions such as pullbacks, which exist but can not be chosen in a canonical and coherent way).

The other natural choice would be to directly use the identity type on the set of $U$-setoids, in a direct type-theoretic formalisation. This would probably end up both formally and conceptually more complicated, but the author intends to explore this possibility in future research.

Before we can go on, we must verify that the equality relation chosen is reflexive, symmetric and transitive.

- Reflexivity is no problem, since taking the standard reflexivity proof for the underlying $U$-element makes the second part trivial.

- For symmetry, the assumption $\mathcal{A}=\mathcal{B}$ gives us a proof $\alpha: \operatorname{ld}_{U}(A, B)$, and standard treatment of identity types yields a proof $\alpha^{-1}: \operatorname{ld}_{U}(B, A)$. We also have a proof that $(\forall x, y: A)\left(x={ }_{\mathcal{A}} y \longleftrightarrow \operatorname{subst}_{\alpha}(x)={ }_{\mathcal{B}} \operatorname{subst}_{\alpha}(y)\right)$. As a particular instance of this, we have

$$
\operatorname{subst}_{\alpha^{-1}}(x)={ }_{\mathcal{A}} \operatorname{subst}_{\alpha^{-1}}(y) \longleftrightarrow \operatorname{subst}_{\alpha}\left(\operatorname{subst}_{\alpha^{-1}}(x)\right)={ }_{\mathcal{B}} \operatorname{subst}_{\alpha}\left(\operatorname{subst}_{\alpha^{-1}}(y)\right) \text {, }
$$

and since $\operatorname{ld}_{B}\left(x\right.$, subst $_{\alpha}\left(\right.$ subst $\left.\left._{\alpha^{-1}}(x)\right)\right)$ holds generally, we are done.

- For transitivity, the idea is again similar: from the assumptions $\mathcal{A}=\mathcal{B}$ and $\mathcal{B}=\mathcal{C}$ we get proofs $\alpha: \operatorname{ld}_{U}(A, B)$ and $\beta: \operatorname{ld}_{U}(B, C)$, and the standard treatment of identity types gives us not only $\beta \circ \alpha: \operatorname{ld}_{U}(A, C)$ but also the identity $\operatorname{Id}_{C}\left(\operatorname{subst}_{\beta}\left(\operatorname{subst}_{\alpha}(x)\right)\right.$, subst so $\left.\alpha(x)\right)$, and we can reason as for symmetry.

The setoid of $U$-setoids just constructed will be the interpretation of the sort $C_{0}$ of objects of the category.

\section{SETOID MAPS IN $U$}

The basic sense of setoid maps $\mathcal{A} \rightarrow \mathcal{B}$ is that they are functions $A \rightarrow B$, between the underlying sets, satisfying an extensionality condition, as mentioned earlier. For $U$-setoids, a map $f: \mathcal{A} \rightarrow \mathcal{B}$ is an element of a $\Sigma$-set, consisting of

- a function $f: T(A) \rightarrow T(B)$, together with

- a proof ext $f:(\forall x, y: T(A))\left(T\left(x={ }_{\mathcal{A}} y\right) \longrightarrow T\left(f(x)={ }_{\mathcal{B}} f(y)\right)\right)$ that this function respects the equality relations on $\mathcal{A}$ and $\mathcal{B}$.

As in the definition of a $U$-setoid, stronger assumptions on $U$ would allow a less cluttered notation.

To prove that two maps $f, g: \mathcal{A} \rightarrow \mathcal{B}$ are equal we must show that

$$
(\forall x: T(A))\left(T\left(f(x)={ }_{\mathcal{B}} g(x)\right)\right),
$$

that is, provide a proof that they are pointwise equal. Showing that this defines a setoid of maps $\mathcal{A} \rightarrow \mathcal{B}$ is easy.

However, this is only a particular homset, and the particular feature of a small category is that we have a single sort for all arrows (and work in a standard manysorted first order theory, rather than a dependently sorted one). This means that all the $U$-setoid maps must form a single setoid. Before defining this setoid, let us see how maps between $U$-setoids and equality of $U$-setoids fit together. 
Let us first note that given two elements $A, B: U$ of the universe and an identity proof $\alpha: \operatorname{ld}_{U}(A, B)$ the substitution operation subst ${ }_{\alpha}$ is a function $T(A) \rightarrow T(B)$. Then, if we have a proof $\alpha: \mathcal{A}=\mathcal{B}$ of an equality of $U$-setoids, we can extract an identity proof $\bar{\alpha}: \operatorname{ld}_{U}(A, B)$ from it, and a corresponding function subst $\bar{\alpha}$. Then the content of the remaining part of the proof object $\alpha$ is that this function is an injective map $\mathcal{A} \rightarrow \mathcal{B}$ of $U$-setoids. Moreover, it is easy to verify that the proof $\alpha^{-1}: \mathcal{B}=\mathcal{A}$, obtained by symmetry of equality, yields a map subst $\overline{\alpha^{-1}}$ which is an inverse for the map subst $\alpha$. Also, given equality proofs $\alpha: \mathcal{A}=\mathcal{B}$ and $\beta: \mathcal{B}=\mathcal{C}$, transitivity of equality yields a proof $\beta \circ \alpha: \mathcal{A}=\mathcal{C}$, and in fact

$$
\operatorname{subst}_{\overline{\beta \circ \alpha}}=\operatorname{subst}_{\bar{\beta}} \circ \text { subst }_{\bar{\alpha}}
$$

as maps of $U$-setoids. This makes it natural to let the isomorphism subst $\bar{\alpha}_{\bar{\alpha}}$ of $U$ setoids obtained from an equality proof $\alpha$ also be denoted by $\alpha$, and no confusion should arise.

Now, with this preparation done, we are ready to define our setoid $C_{1}$ of arrows. The underlying set is a $\Sigma$-set, whose elements are tuples consisting of

- two $U$-setoids $\mathcal{A}$ and $\mathcal{B}$, together with

- a map $f: \mathcal{A} \rightarrow \mathcal{B}$.

Since the $U$-setoids form a set $C_{0}$ : Set, as do the maps, this set can be formed. In contrast, since the setoids do not form a set a similar construction can not be carried out for them. This is the point where the use of a universe is necessary.

Next, we must define an equality. A proof that two arrows $f: \mathcal{A} \rightarrow \mathcal{B}$ and $g: \mathcal{C} \rightarrow \mathcal{D}$ are equal consist of

- two equality proofs $\alpha: \mathcal{A}=\mathcal{C}$ and $\beta: \mathcal{D}=\mathcal{B}$, for domains and codomains (note the order of the codomains - equality proofs are directed, so this matters); together with

- a proof that $f=\beta \circ g \circ \alpha$, as $U$-setoid maps $\mathcal{A} \rightarrow \mathcal{B}$.

This can also be put as having two equality proofs $\alpha$ and $\beta$ as above, together with a proof that the diagram

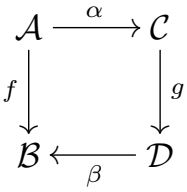

commutes, where we write $\alpha$ and $\beta$ not only for the proofs of equality, but also for the substitution maps derived from them.

For the arrows to form a setoid, we must also verify that the equality relation is an equivalence relation:

- Proving reflexivity is easy, since using the standard reflexivity proofs on the $U$-setoids makes $\alpha$ and $\beta$ both be the identity map.

- For symmetry, we of course use the symmetry of setoid equalities. Then note that if $f=\beta \circ g \circ \alpha$, then also $\beta^{-1} \circ f \circ \alpha^{-1}=\beta^{-1} \circ(\beta \circ g \circ \alpha) \circ \alpha^{-1}$ (since composition of maps respects equality). But the latter equals $g$, by our preparatory results. 
- Finally, transitivity is easy, considering the diagram

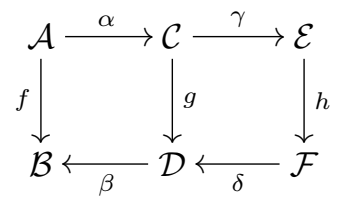

and earlier observations.

We should note that given a propositional equality on the $U$-setoids, there is essentially only one choice for the equality on arrows (apart from cosmetic differences such as taking equality proofs of $U$-setoids in the other direction in the diagram above).

\section{Composable Setoid maps in $U$}

The third, and last, sort in the theory of a category is that of composable arrows. It is not immediately clear what the best choice is for the setoid $C_{2}$ of composable pairs. There are a few constraints to take into account though: The setoid $C_{2}$ should have two projection functions fst, snd: $C_{2} \rightarrow C_{1}$, and not only should these respect equality, Axiom 5 also tells us that

$$
\operatorname{fst}(u)=\operatorname{fst}(v) \& \operatorname{snd}(u)=\operatorname{snd}(v) \longrightarrow u=v,
$$

so these projections actually define the equality on $C_{2}$. We must also consider Axiom 6, which says that if $\operatorname{dom}(f)=\operatorname{cod}(g)$, then $g$ and $f$ form a composable pair.

With this in mind, there seems to be two natural choices. The first one would be to take $C_{2}$ to consist of pairs $\langle g, f\rangle$ of arrows together with a proof that $\operatorname{dom}(f)=$ $\operatorname{cod}(g)$. The main reason not to choose this version is that it introduces more equality proofs into the constructions. There is also another problem, namely that the composition operation would have to send $\langle\langle g, f\rangle, \alpha\rangle$ to $f \circ \alpha \circ g$, and thus two composable pairs differing only in the equality proof could be composed to different arrows. However, it appears that this problem is unavoidable.

The other choice, which seems easier to work with, is to take $C_{2}$ to consist of diagrams of the shape $\longrightarrow \longrightarrow \cdot$, or in other words, $C_{2}$ is the $\Sigma$-set whose elements consist of

- three $U$-setoids $\mathcal{A}, \mathcal{B}$, and $\mathcal{C}$; together with

- two maps $f: \mathcal{A} \rightarrow \mathcal{B}$ and $g: \mathcal{B} \rightarrow \mathcal{C}$.

The projection functions are defined by

$$
\begin{aligned}
& \text { fst }: \mathcal{A} \stackrel{f}{\longrightarrow} \mathcal{B} \stackrel{g}{\longrightarrow} \mathcal{C} \mapsto \mathcal{A} \stackrel{f}{\longrightarrow} \mathcal{B} \text { and } \\
& \text { snd: } \mathcal{A} \stackrel{f}{\longrightarrow} \mathcal{B} \stackrel{g}{\longrightarrow} \mathcal{C} \mapsto \mathcal{B} \stackrel{g}{\longrightarrow} \mathcal{C}
\end{aligned}
$$

(and as the notation suggests, this also gives the interpretation of these function symbols).

As indicated earlier, equality is then given by

$$
u=v \longleftrightarrow \mathrm{fst}(u)=\mathrm{fst}(v) \& \operatorname{snd}(u)=\operatorname{snd}(v) .
$$

It is trivial to verify that this relation is reflexive, symmetric and transitive.

While it may seem that this definition avoids the second problem indicated earlier, this is not in fact the case, as we will see later. 


\section{INTERPRETING THE FUNCTION SYMBOLS}

As we have now provided interpretations for all three sorts, it is time to provide interpretations for the six function symbols. These interpretations should be setoid maps between the appropriate sort interpretations, so we must not only define the functions, but also verify that the functions are extensional, that is, that they respect the setoid equalities.

- id: $C_{0} \rightarrow C_{1}$ should give the identity arrow on an object, so in our case we send a $U$-setoid $\mathcal{A}$ to its identity $1_{\mathcal{A}}$, which is of course given by the identity function, and is easily shown to be a map of $U$-setoids.

To see that this function is extensional, suppose $\alpha: \mathcal{A}=\mathcal{B}$ is a proof of equality of $U$-setoids. That id is extensional then follows from the commutativity of the diagram

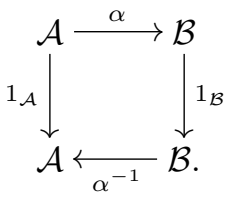

- dom: $C_{1} \rightarrow C_{0}$ and cod: $C_{1} \rightarrow C_{0}$ should give the domain and codomain of an arrow. In our case, these are simple projection functions, since the domain and codomain $U$-setoids are explicitly part of an arrow.

It is also trivially extensional, since proofs of equalities between domains and between codomains are part of a proof of equality of arrows.

- fst: $C_{2} \rightarrow C_{1}$ and snd: $C_{2} \rightarrow C_{1}$ should give the two arrows of a composable pair. These were already defined above, and the definition of the equality on $C_{2}$ automatically makes them extensional.

- comp: $C_{2} \rightarrow C_{1}$ should send a composable pair to its composite arrow. In our case, we naturally send the composable pair $\mathcal{A} \stackrel{g}{\longrightarrow} \mathcal{B} \stackrel{f}{\longrightarrow}$ to $\mathcal{A} \stackrel{f \circ g}{\longrightarrow} \mathcal{C}$.

For extensionality, suppose we have two composable pairs $u$ and $v$, and a proof that $u=v$, that is, we have equalities $\alpha, \beta, \gamma$, and $\delta$ of $U$-setoids, and proofs that the left and right square in the diagram

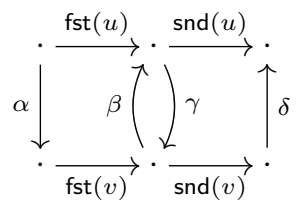

commute. Note that $\gamma \circ \beta$ is a reflexivity proof for a $U$-setoid. It then follows from the principle US-refl $(U)$ that the corresponding map $\gamma \circ \beta$ equals the identity map (since it is pointwise Id-equal to the identity). Thus we have $\operatorname{comp}(u)=\operatorname{snd}(u) \circ \mathrm{fst}(u)=\delta \circ \operatorname{snd}(v) \circ \gamma \circ \beta \circ \mathrm{fst}(v) \circ \alpha=\delta \circ \operatorname{snd}(v) \circ \mathrm{fst}(v) \circ \alpha=$ $\delta \circ \operatorname{comp}(v) \circ \alpha$, which provides the required extensionality.

\section{INTERLUDE: THE PRINCIPLE US-REFL}

In the previous section the principle US-refl $(U)$ was used to prove the extensionality of the composition function comp: $C_{2} \rightarrow C_{1}$. This section has two goals: first, 
to show that the extensionality of comp is unprovable without additional principles; and second, to clarify how the principle US-refl relates to the principle UIP of uniqueness of identity proofs.

For the unprovability result, consider the groupoid model of type theory with a universe interpreted by the groupoid of $V$-small groupoids - together with their isomorphisms - as in [6]. The propositional-equality-is-isomorphism model discussed at the end of that paper gives a helpful intuition for a counter-example.

Let us particularly pick out three elements of this universe: the six-element set $\{1,2,3,4,5,6\}$ (viewed as a discrete groupoid), $\{1,2,3\}$, and $\{1,2\}$. Together with their respective identity types these form $U$-setoids. Note that the model construction gives us the full symmetric groups as the collections of reflexivity proofs of these sets, and that, trivially, these extend to equalities of $U$-setoids. In particular, consider the identity proof corresponding to the cyclic permutation $(123)$ on $\{1,2,3\}$, and particularly note that $(123)(123)=(132)$. Also consider the functions $f:\{1,2,3,4,5,6\} \rightarrow\{1,2,3\}$ and $g:\{1,2,3\} \rightarrow\{1,2\}$ given by

$$
\left\{\begin{array} { l } 
{ f ( 1 ) = f ( 2 ) = f ( 3 ) = 1 , } \\
{ f ( 4 ) = f ( 5 ) = 2 , } \\
{ f ( 6 ) = 3 }
\end{array} \quad \text { and } \quad \left\{\begin{array}{l}
g(1)=g(2)=1, \\
g(3)=2 .
\end{array}\right.\right.
$$

The diagram

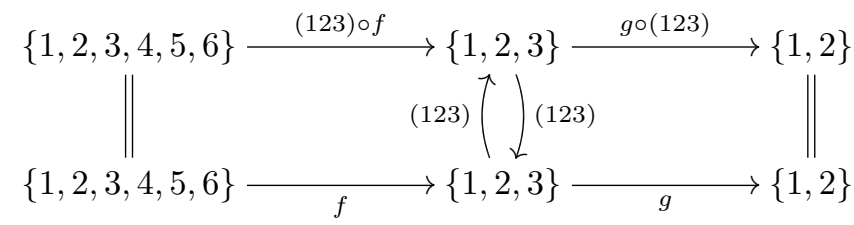

then exhibits two equal composable pairs. But now note that

- the composition of the upper pair is $g \circ(132) \circ f$ which sends $\{4,5\}$ to 1 and $\{1,2,3,6\}$ to 2 ; while

- the composition of the lower pair is $g \circ f$ which sends $\{1,2,3,4,5\}$ to 1 and $\{6\}$ to 2 .

Mere counting makes it clear that it is impossible to conjugate by permutations on $\{1,2,3,4,5,6\}$ and $\{1,2\}$ (that is, reflexivity proofs on the domain and codomain) to exhibit these as equal arrows. But that means the composition function is not extensional in this model, and hence the extensionality of comp is unprovable.

While the counterexample above is quite straight-forward, there is a subtlety worth mentioning: if we also have an elimination rule for the universe $U$, as outlined in [9, Chapter 14], then any sufficiently "simple" ${ }^{2} U$-set $u$ makes $[x: U] \operatorname{ld}_{U}(u, x)$ a decidable relation, that is $(\forall x: U)\left(\operatorname{Id}_{U}(u, x) \vee \neg \mathbf{d}_{U}(u, x)\right)$, and hence locally there are unique identity proofs (see [12], strengthening the results of [4]), and in particular $\left(\forall \alpha: \operatorname{Id}_{U}(u, u)\right) \operatorname{Id}_{\mathbf{I d}_{U}(u, u)}(\alpha, \mathrm{r}(u))$. That means that simple $U$-sets have no nontrivial automorphism - but the counterexample above depends on such. Hence, in the presence of a universe elimination rule, any counterexample must arise as the interpretation of "complicated" types (essentially, as dependent types).

\footnotetext{
${ }^{2}$ The typical simple $U$-sets are those named by constructors of $U$, as true, false, and the natural numbers, but some further constructions like the disjoint sum are also simple enough.
} 
Having shown that some additional principle must be added to type theory, let us go back to the principle suggested and used:

(US-refl $(U)$ )

$$
X: U, \alpha: \operatorname{ld}_{U}(X, X), x: T(X) \vdash \operatorname{Id}_{T(X)}\left(x, \operatorname{subst}_{\alpha}(x)\right) .
$$

For comparison, let us also consider the following principles:

$$
\begin{aligned}
& \mathrm{US}(U) \quad X, Y: U, \alpha, \beta: \mathrm{Id}_{U}(X, Y), x: T(X) \vdash \operatorname{Id}_{T(Y)}\left(\operatorname{subst}_{\alpha}(x), \operatorname{subst}_{\beta}(x)\right) \\
& \mathrm{UIP}-\operatorname{refl}(U) \quad X: U, \alpha: \operatorname{ld}_{U}(X, X) \vdash \operatorname{ld}_{\mathbf{l d}_{U}(X, X)}(\alpha, \mathrm{r}(X)) \\
& \operatorname{UIP}(U) \quad X, Y: U, \alpha, \beta: \operatorname{Id}_{U}(X, Y) \vdash \operatorname{Id}_{\operatorname{ld}_{U}(X, Y)}(\alpha, \beta) \\
& \text { UIP } \quad A: \text { Set, } a, b: A, \alpha, \beta: \operatorname{Id}_{A}(a, b) \vdash \operatorname{Id~}_{\mathbb{I d}_{A}(a, b)}(\alpha, \beta) \\
& \mathrm{US}^{+}(U) \quad C:(U) \text { Set, } X, Y: U, \alpha, \beta: \operatorname{ld}_{U}(X, Y), x: C(X) \\
& \vdash \mathrm{Id}_{C(Y)}\left(\operatorname{subst}_{\alpha, C}(x), \text { subst }_{\beta, C}(x)\right) \\
& \text { US } \quad A: \text { Set, } C:(A) \text { Set, } a, b: A, \alpha, \beta: \operatorname{ld}_{A}(a, b), x: C(a) \\
& \vdash \operatorname{Id}_{C(b)}\left(\operatorname{subst}_{\alpha, C}(x) \text {, subst } \beta, C(x)\right)
\end{aligned}
$$

The last three principles are generalisations of earlier principles; note particularly that $\mathrm{US}^{+}(U)$ generalises from substitutions specifically in the universe-decoding family $T$ to arbitrary families, and the full principle US further to arbitrary sets.

Proposition 1. The following implications hold between the principles mentioned above:

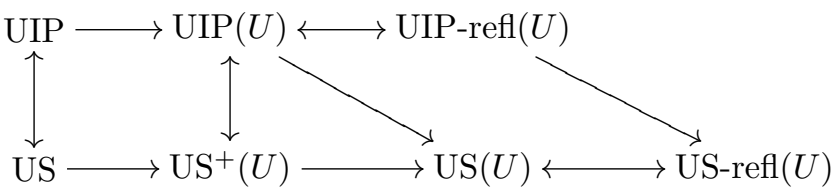

Proof. The implications in the top line are trivial or well-known. Similarly, the implications in the bottom line are easy. For completeness, let us consider the implication US-refl $(U) \longrightarrow \mathrm{US}(U)$ : assume US-refl $(U)$ and suppose we have two identity proofs $\alpha, \beta: \operatorname{ld}_{U}(X, Y)$. Then $\beta^{-1} \circ \alpha: \operatorname{ld}_{U}(X, X)$, so subst $\beta^{-1} \circ \alpha$ is pointwise equal to the identity, by US-refl $(U)$. But we also know that $\alpha={ }_{\mathrm{ld}} \beta \circ\left(\beta^{-1} \circ \alpha\right)$, so it follows that subst ${ }_{\alpha}={ }_{p t}$ subst $_{\beta \circ\left(\beta^{-1} \circ \alpha\right)}={ }_{p t}$ subst $_{\beta} \circ$ subst $_{\beta^{-1} \circ \alpha}={ }_{p t}$ subst $_{\beta} \circ$ subst $_{\mathrm{r}(X)}={ }_{p t}$ subst $_{\beta}$ as required.

All the top-to-bottom implications are easy, and follow immediately by elimination of the identity proof provided by the corresponding version of UIP.

Next, consider the implication US $\longrightarrow$ UIP. Rather than showing this implication directly, let us show that US implies the principle

$$
A \text { : Set, } a: A, \alpha: \operatorname{Id}_{A}(a, a) \vdash \operatorname{Id}_{\operatorname{Id}_{A}(a, a)}(\alpha, \mathrm{r}(a)),
$$

which is clearly equivalent to UIP. So suppose US is valid, and take $A$ : Set, $a: A$, and $\alpha: \operatorname{ld}_{A}(a, a)$ arbitrary. We have of course that $\alpha \circ \mathrm{r}(a)=\mathrm{ld} \alpha$. But note that $\alpha \circ \mathrm{r}(a)$ is, by definition, subst $\alpha,[x: A] \operatorname{ld}_{A}(a, x)(\mathrm{r}(a))$, and hence US (or even the obvious equivalent US-refl) gives us $\alpha \circ \mathrm{r}(a)=\operatorname{subst}_{\alpha,[x: A] \operatorname{ld}_{A}(a, x)}(\mathrm{r}(a))={ }_{\operatorname{ld}}$ $\operatorname{subst}_{\mathrm{r}(a),[x: A] \operatorname{ld}_{A}(a, x)}(\mathrm{r}(a))=\mathrm{r}(a)$, with the first and last equalities definitional, as required.

Finally, note that in the preceding proof, if we only consider the case $A=U$, that is, we prove the principle UIP-refl $(U)$, then the instance of US used is actually an instance of $\mathrm{US}^{+}(U)$, so this also proves the implication $\mathrm{US}^{+}(U) \longrightarrow \mathrm{UIP}(U)$.

Proposition 2. The implications $\mathrm{US}(U) \longrightarrow \mathrm{UIP}(U), \mathrm{US}(U) \longrightarrow \mathrm{US}^{+}(U)$, and US$\operatorname{refl}(U) \longrightarrow \mathrm{UIP}-\mathrm{refl}(U)$ are not provable. 
Proof. Given the implications shown in Proposition 1, it is enough to show that one of these implications is not provable.

Let us again consider the groupoid model of type theory. The standard interpretation of the universe $U$ would be the groupoid $G p d(V)$ of $V$-small groupoids and their isomorphisms, for some metatheoretic universe $V$. The decoding family would be given by the (full and faithful) inclusion functor into the category Gpd of groupoids in the (larger) metatheoretic universe $\mathcal{V}$ interpreting Set. By modifying the interpretations of $U$ and $T$, we will find a model where the implications fail.

The basic intuition is that if we restrict the morphisms in $U$ to those automorphisms which leave the connected components of the groupoids invariant (though not necessarily pointwise fixed), then $\operatorname{UIP}(U)$ fails since there are many parallel such functors, while there is always an arrow from an object to its image under such a functor, loosely corresponding to US-refl $(U)$. To actually be able to interpret a term in the type given by $\operatorname{US}-\operatorname{refl}(U)$, this needs to be elaborated.

In the general case, the universe $U$ will be interpreted by a small groupoid $U \in G p d$, and the decoding family $T$ by a functor $T: U \rightarrow G p d$. Using the interpretation of type theory in the groupoid model according to [6], we interpret the principle US-refl $(U)$.

- The context $X: U, \alpha: \operatorname{ld}_{U}(X, X), x: T(X)$ is interpreted by a groupoid $\Gamma$ whose

- objects are triples $(X, \alpha, x)$ where $X \in U, \alpha: X \rightarrow X$ in $U$, and $x \in T(X)$; and whose

- arrows $(X, \alpha, x) \rightarrow\left(X^{\prime}, \alpha^{\prime}, x^{\prime}\right)$ are triples $(q, r, s)$ where $q: X \rightarrow X^{\prime}$ in $U$ is such that $q \circ \alpha \circ q^{-1}=\alpha^{\prime}, r=*$ witnessing the previous equality $\left(r\right.$ is an arrow in a discrete category), and $s: T(q)(x) \rightarrow x^{\prime}$ in $T\left(X^{\prime}\right)$.

- The type (or family of sets) $\operatorname{Id}_{T(X)}\left(x, \operatorname{subst}_{\alpha}(x)\right)$ is interpreted by a functor $\Gamma \rightarrow$ Gpd assigning

- to an object $(X, \alpha, x) \in \Gamma$ the discrete category $\Delta(T(X)(x, T(\alpha)(x)))$, that is the set of arrows $x \rightarrow T(\alpha)(x)$ in $T(X)$;

- to an arrow $(q, r, s):(X, \alpha, x) \rightarrow\left(X^{\prime}, \alpha^{\prime}, x^{\prime}\right)$ in $\Gamma$ the function (functor between discrete categories) $T(X)(x, T(\alpha)(x)) \rightarrow T\left(X^{\prime}\right)\left(x^{\prime}, T\left(\alpha^{\prime}\right)\left(x^{\prime}\right)\right)$ sending an arrow $t: x \rightarrow T(\alpha)(x)$ in $T(X)$ to the arrow

$$
T\left(\alpha^{\prime}\right)(s) \circ T(q)(t) \circ s^{-1}: x^{\prime} \rightarrow T\left(\alpha^{\prime}\right)\left(x^{\prime}\right)
$$

in $T\left(X^{\prime}\right)$. It is clear that this function is invertible, so it provides an arrow in $G p d$.

To provide a dependent object of this family is to provide

- for every object $(X, \alpha, x) \in \Gamma$ an arrow $\mu_{(X, \alpha, x)}: x \rightarrow T(\alpha)(x)$ in $T(X)$; and

- for every arrow $(q, r, s):(X, \alpha, x) \rightarrow\left(X^{\prime}, \alpha^{\prime}, x^{\prime}\right)$ in $\Gamma$ an arrow

$$
T\left(\alpha^{\prime}\right)(s) \circ T(q)\left(\mu_{(X, \alpha, x)}\right) \circ s^{-1} \rightarrow \mu_{\left(X^{\prime}, \alpha^{\prime}, x^{\prime}\right)}
$$

in $\Delta\left(T\left(X^{\prime}\right)\left(x^{\prime}, T\left(\alpha^{\prime}\right)\left(x^{\prime}\right)\right)\right)$. In other words, the equality

$$
T\left(\alpha^{\prime}\right)(s) \circ T(q)\left(\mu_{(X, \alpha, x)}\right) \circ s^{-1}=\mu_{\left(X^{\prime}, \alpha^{\prime}, x^{\prime}\right)}
$$

must hold.

We aim to find a category $U$ and functor $T: U \rightarrow G p d$ where such a dependent object exists, since it would provide an interpretation for a (new) term witnessing 
US-refl $(U)$. As a first step, we consider a slightly simpler sufficient condition, and then construct examples where this simpler condition holds.

Suppose the category $U$ is such that every arrow in $U$ is an automorphism. Suppose further that for every arrow $f: X \rightarrow X$ in $U$ there is a chosen natural transformation $\phi(f): \mathrm{id}_{T(X)} \rightarrow T(f)$ satisfying $\phi\left(f^{-1}\right)_{z}=\phi(f)_{f^{-1}(z)}^{-1}$ and $\phi(f \circ g)=\phi(f) * \phi(g)$ (the horizontal composition of natural transformations). Choosing $\mu_{(X, \alpha, x)}=\phi(\alpha)_{x}$ for the dependent object, these equalities, together with the naturality of both $\phi(\alpha)$ and $\phi(q)$, suffice for proving the equality (1) above.

It then only remains to provide $U$ and $T$ satisfying these conditions. Let us provide two instances: first a slightly simpler one, but which restricts what small groupoids are included in $U$; then a slightly more complicated one, but which allows us to include all $V$-small groupoids as objects of $U$.

For the first case, take $U$ to have as objects those $V$-small groupoids where any parallel arrows are equal, and as arrows those automorphisms $f: X \rightarrow X$ such that there is a natural transformation $\mathrm{id}_{X} \rightarrow f$. It is easily verified that this is a groupoid. Further, take $T$ to be the inclusion of $U$ into Gpd. Since any parallel arrows in any object of $U$ are equal, it follows that any parallel natural transformations are equal, and the conditions above hold. This thus provides a model where US-refl $(U)$ holds. But note that there are nontrivial automorphisms in $U$ - for example, consider the groupoid having two uniquely isomorphic objects; then the automorphism exchanging the two objects satisfies the condition above so UIP-refl $(U)$ fails.

For the second case, take $U$ to have as objects all $V$-small groupoids, but as arrows pairs $(f, \bar{f}): X \rightarrow X$ where $f: X \rightarrow X$ is an automorphism on the $V$-small groupoid $X$, and $\bar{f}$ is a natural transformation $\mathrm{id}_{X} \rightarrow f$ (and all arrows are of this form). Identities are given by the pairs $\left(\mathrm{id}_{X}, \mathrm{id}_{\mathrm{id}_{X}}\right)$, composition by $(f, \bar{f}) \circ(g, \bar{g})=$ $(f \circ g, \bar{f} * \bar{g})$, and inverses by $(f, \bar{f})^{-1}=\left(f^{-1}, \overline{f^{-1}}\right)$, where the component at $x \in X$ of $\bar{f}^{-1}$ is the inverse of $\bar{f}_{f^{-1}(x)}$. It is easily verified that this is a groupoid. The functor $T: U \rightarrow G p d$ is identity on objects, and first projection on arrows. Again, the conditions above are easily verified, so this provides another model where US-refl $(U)$ holds, and again we note that there are nontrivial automorphisms in $U$ (for example the one mentioned previously, together with the obvious natural transformation), so UIP-refl $(U)$ fails.

To directly see that the model above does not satisfy $\mathrm{US}^{+}(U)$, consider the small groupoid $G$ of shape

$$
a \quad b \quad c \stackrel{\cong}{\longrightarrow} d
$$

and note that there are two $U$-automorphisms on $G$ : the identity and the automorphism $f$ fixing $a$ and $b$ and swapping $c$ and $d$. Now, construct a functor $F: U \rightarrow G p d$ (that is, a Set-valued family over $U$ ) acting as the inclusion everywhere except at $G$. Let $F(G)=G$, but send the $U$-automorphism $f$ to the automorphism $f^{\prime}$ also swapping $a$ and $b$. Since $f^{\prime} \circ f^{\prime}=i d=f \circ f$, functoriality holds. Now, by a calculation similar to the one in Proposition 2, subst ${ }_{f, F}(a)=F(f)(a)=f^{\prime}(a)=b$, while subst $\operatorname{r}_{(G), F}(a)=F\left(\operatorname{id}_{G}\right)(a)=\operatorname{id}_{G}(a)=a$, and hence, for $\mathrm{US}^{+}(U)$ to hold, we must have an arrow $a \rightarrow b$ in $G$. This shows that the family $F$ refutes $\operatorname{US}^{+}(U)$.

Finally, note that the implications $\mathrm{US}^{+}(U) \longrightarrow \mathrm{US}$ and $\operatorname{UIP}(U) \longrightarrow$ UIP are not provable either, as evidenced by the interpretation of $U$ as the discrete groupoid of $V$-small groupoids (as done in [5, p. 146]). 
We have thus shown that the principle US-refl $(U)$, sufficient for the constructions studied, is strictly weaker than the corresponding principle of uniqueness of identity proofs $\operatorname{UIP}(U)$. There are still questions remaining open: Is extensionality of the function comp equivalent to US-refl $(U)$, or if not, is there a principle of independent interest which is necessary and sufficient for the extensionality of comp? Also, what collections of set formers can a universe $U$ be closed under, while satisfying $\operatorname{US}(U)$ but not $\operatorname{UIP}(U)$ ? (Note that the counterexamples constructed above are not closed under $\Sigma$-formation in nonempty contexts.)

\section{VERIFYING THE AXIOMS}

Before the interlude, we provided the interpretations for both sorts and symbols of the first order theory of a small category. To finish, we must also verify that the axioms are satisfied. It is well known that the $U$-setoids and their maps form a so-called E-category (where there is not a single set of arrows, but rather separate homsets, and a whole family of composition functions), and we will make use of this, particularly in the diagrams below.

(1) $\operatorname{dom}(\operatorname{id}(x))=x$-immediate, since the equality holds definitionally.

(2) $\operatorname{cod}(\operatorname{id}(x))=x-\operatorname{ditto}$.

(3) $\operatorname{dom}(\operatorname{comp}(u))=\operatorname{dom}(\operatorname{fst}(u))-\operatorname{ditto}$.

(4) $\operatorname{cod}(\operatorname{comp}(u))=\operatorname{cod}(\operatorname{snd}(u))-$ ditto.

(5) $\operatorname{fst}(u)=\operatorname{fst}(v) \& \operatorname{snd}(u)=\operatorname{snd}(v) \longrightarrow u=v$ - by definition of the equality in $C_{2}$.

(6) $\operatorname{dom}(f)=\operatorname{cod}(g) \longrightarrow\left(\exists u \in C_{2}\right)($ fst $(u)=g \& \operatorname{snd}(u)=f)$ - suppose we are given arrows $f$ and $g$ and a proof $\alpha$ : $\operatorname{dom}(f)=\operatorname{cod}(g)$. Then the following diagram provides the required composable pair $u$ as the top line, and the commutativity of the two squares proves the required equalities:

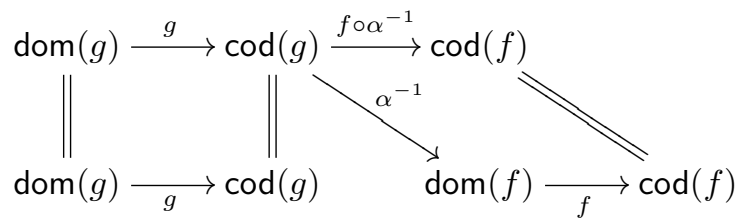

where of course the intended proofs for the vertical equalities are the standard reflexivity proofs.

(7) $f \sim f \circ \operatorname{id}(\operatorname{dom}(f))$ - unfolding the shorthand notation, we see that we must provide a composable pair $u$ such that $\mathrm{fst}(u)=\mathrm{id}(\operatorname{dom}(f))$, snd $(u)=f$, and $\operatorname{comp}(u)=f$. Now, using Axiom 2 we have $\operatorname{cod}(\operatorname{id}(\operatorname{dom}(f)))=\operatorname{dom}(f)$ (as witnessed by the standard reflexivity proof), so we can consider the composable pair constructed for the verification of Axiom 6. This only leaves the final equation $\operatorname{comp}(u)=f$ to be verified, but inspection of the construction shows that $\operatorname{comp}(u)=f \circ$ id $\circ$ id, so this is easy.

(8) $f \sim \operatorname{id}(\operatorname{cod}(f)) \circ f-$ similar to the previous.

(9) $k \sim f \circ g \& l \sim g \circ h \longrightarrow k \circ h \sim f \circ l$ - the associativity axiom looks innocuous, but turns out to be surprisingly complicated. Unfolding the shorthands in 
the assumptions, we get the given data indicated in the following diagram:

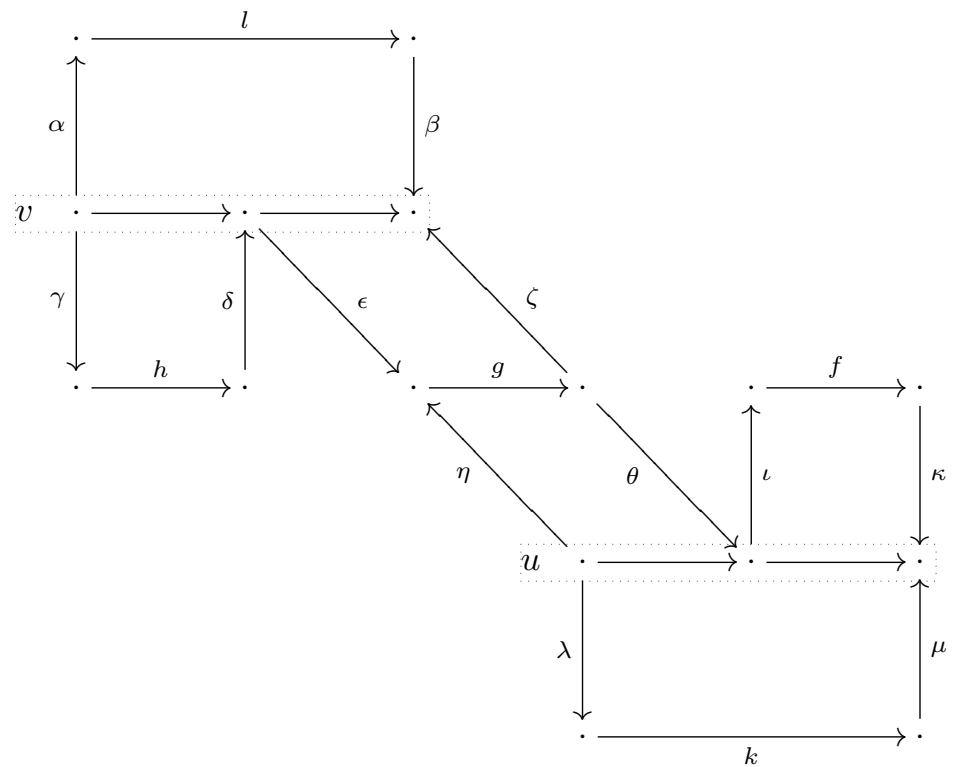

The boxes indicate the given composable pairs, and the small Greek letters indicate proofs of $U$-setoid equalities.

Unfolding shorthands in the consequence, we see that we must provide an arrow $m$, and two composable pairs $w$ and $z$ whose composites equal $m$ (one for $k$ and $h$, and one for $f$ and $l$ ). These, and the required equality proofs are indicated in the diagram below.

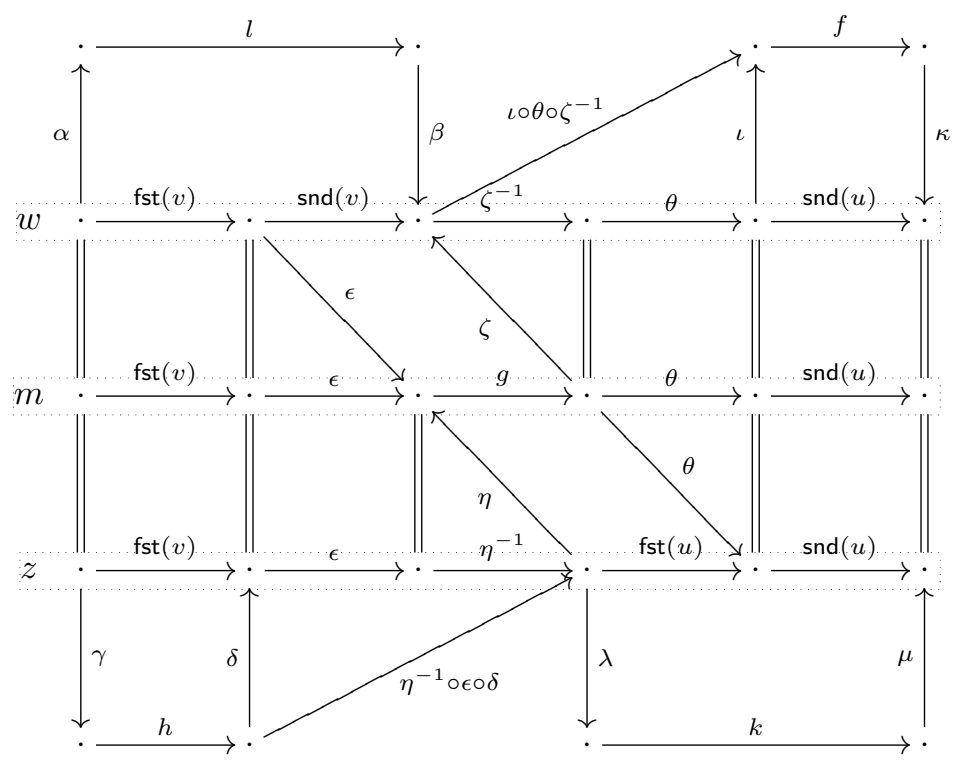

Note that this diagram is pasted together from (commutative) parts of the previous, and some trivially commutative parts, and so is itself a commutative diagram. Also note that the commutativity of this diagram has 
not required another use of US-refl$(U)$, but actually holds directly in the E-category of $U$-setoids.

Thus, all required axioms hold, and we have exhibited a small category of setoids.

\section{REFERENCES}

[1] E. Bishop. Foundations of constructive analysis. McGraw-Hill Book Co., New York, 1967.

[2] E. Bishop and D. Bridges. Constructive analysis, volume 279 of Grundlehren der Mathematischen Wissenschaften [Fundamental Principles of Mathematical Sciences]. Springer-Verlag, Berlin, 1985.

[3] D. Fridlender. A proof-irrelevant model of Martin-Löf's logical framework. Math. Structures Comput. Sci., 12(6):771-795, 2002.

[4] M. Hedberg. A coherence theorem for Martin-Löf's type theory. J. Funct. Programming, 8(4):413-436, 1998.

[5] M. Hofmann. Extensional constructs in intensional type theory. CPHC/BCS Distinguished Dissertations. Springer-Verlag London Ltd., London, 1997.

[6] M. Hofmann and T. Streicher. The groupoid interpretation of type theory. In Twenty-five years of constructive type theory (Venice, 1995), volume 36 of Oxford Logic Guides, pages 83-111. Oxford Univ. Press, New York, 1998.

[7] P. T. Johnstone. Sketches of an elephant: a topos theory compendium. Vol. 1, volume 43 of Oxford Logic Guides. The Clarendon Press Oxford University Press, New York, 2002.

[8] S. Mac Lane. Categories for the Working Mathematician, volume 5 of Graduate Texts in Mathematics. Springer-Verlag, New York, second edition, 1998.

[9] B. Nordström, K. Petersson, and J. M. Smith. Programming in Martin-Löf's type theory, volume 7 of International Series of Monographs on Computer Science. The Clarendon Press Oxford University Press, New York, 1990. An introduction.

[10] B. Nordström, K. Petersson, and J. M. Smith. Martin-Löf's type theory. In Handbook of logic in computer science, Vol. 5, volume 5 of Handb. Log. Comput. Sci., pages 1-37. Oxford Univ. Press, New York, 2000.

[11] E. Palmgren. Constructivist and structuralist foundations: Bishop's and Lawvere's theories of sets. Technical Report 4, Institut Mittag-Leffler, Sweden, fall 2009.

[12] E. Palmgren. Remarks on the relation between families of setoids and identity in type theory. Technical Report 36, Institut Mittag-Leffler, Sweden, fall 2009.

[13] T. Streicher. Semantical investigations into intensional type theory. Habilitationsschrift, LMU München, 1993. 